\title{
EFFICIENT FITTING OF MULTIPLANET KEPLERIAN MODELS TO RADIAL VELOCITY AND ASTROMETRY DATA
}

\author{
J. T. WRIGHT ${ }^{1}$ AND A. W. HowARD ${ }^{2,3}$ \\ 1226 Space Sciences Building, Astronomy Department, Cornell University, Ithaca, NY 14853, USA; jtwright@ astro.cornell.edu \\ 2601 Campbell Hall, Astronomy Department, University of California, Berkeley, CA 94720, USA \\ Received 2008 October 14; accepted 2009 March 24; published 2009 April 30
}

\begin{abstract}
We describe a technique for solving for the orbital elements of multiple planets from radial velocity (RV) and/or astrometric data taken with $1 \mathrm{~m} \mathrm{~s}^{-1}$ and $\mu$ as precision, appropriate for efforts to detect Earthmassed planets in their stars' habitable zones, such as NASA's proposed Space Interferometry Mission. We include details of calculating analytic derivatives for use in the Levenberg-Marquardt (LM) algorithm for the problems of fitting RV and astrometric data separately and jointly. We also explicate the general method of separating the linear and nonlinear components of a model fit in the context of an LM fit, show how explicit derivatives can be calculated in such a model, and demonstrate the speed up and convergence improvements of such a scheme in the case of a five-planet fit to published RV data for 55 Cnc.
\end{abstract}

Key words: astrometry - methods: data analysis - methods: numerical - planetary systems - techniques: radial velocities

\section{INTRODUCTION}

\subsection{Fitting Keplerian Curves}

The discovery of over 27 multiple-planet systems in recent years (Wright et al. 2009) has required algorithms for disentangling the radial velocity (RV) signature of such complex systems. Because the parameters describing an RV or astrometric curve are nonlinear, there is no way to fit for them analytically, and they must be found through an algorithmic search. Fitting for the Keplerian parameters of a single orbital companion is usually straightforward given a good period guess, and if necessary a "brute force" mapping of the $\chi^{2}$ space is usually not computationally prohibitive. Fitting multiple planets involves searching a correspondingly higher dimensional space and can require substantial computing time.

There is an art to searching such $\chi^{2}$ spaces efficiently, and in this context there are many "tricks" for finding the global minimum. For instance, a Lomb-Scargle periodogram (Scargle 1982 ) is often used to identify promising periods for prospective planets, and all of the tallest peaks can be used as starting guesses for the fitting algorithm. In hierarchical systems, the dominant planet can be fit for alone, its signal subtracted from the data, and additional planets can be searched for among the residuals. This process can then be repeated until all of the planets have been identified, and then a full, multiplanet fit on the original data starting at the values found for the individual planets (e.g., Fischer et al. 2008).

In many multiplanet systems, planet-planet interactions can significantly alter the RV or astrometric signature of the system. In such cases, where interactions are important, a full dynamical (Newtonian) fit involving an $n$-body code must be used to properly fit the data and to ensure the short- and long-term stability of the solution. Even in these cases, a multiplanet Keplerian (kinematic) fit, which simply adds the reflex signatures of single planets and ignores planet-planet interactions, is still useful for efficiently identifying planets and providing good initial guesses to the $n$-body codes.

\footnotetext{
3 Townes Fellow, Space Sciences Laboratory, UC Berkeley, USA.
}

The Levenberg-Marquardt method (LM; Levenberg 1944; Marquardt 1963) is an efficient algorithm for finding a local minimum in a nonlinear $\chi^{2}$ space (given good guesses), and is well suited for the application of RV and astrometric fitting (Press et al. 1992). As a concrete example, we refer in this work to a useful $\mathrm{IDL}^{4}$ implementation of this technique, MPFIT by Craig Markwardt ${ }^{5}$ (Markwardt 2009). Like most implementations of the algorithm, MPFIT requires a user-defined function that accepts, as an argument, trial values for the parameters being solved for, evaluates the model at those values, and returns the corresponding residuals to the data. MPFIT then uses this information to step through parameter space and locate the minimum in $\chi^{2}$ using a combination of Newton's method and a steepest descent method. Uncertainties in these parameters can then be calculated by mapping the $\chi^{2}$ space near this minimum (e.g., Wright et al. 2007) or through error "bootstrapping" (e.g., Butler et al. 2006).

The user-defined function in MPFIT also optionally returns the values of the derivatives of these residuals with respect to the parameters being fit, computed "analytically" or "explicitly." In the absence of these derivatives, MPFIT will calculate numerical derivatives with small steps in the fitted parameters and calculating the resulting change in the residuals. The user must choose step sizes that are not too large-thereby missing fine structure in the $\chi^{2}$ space-or too small-increasing compute time and potentially losing numerical precision. Adding explicit-derivative capability to MPFIT, or any implementation of the LM algorithm, obviates the need for explicitly providing step sizes, and can greatly improve performance in terms of the number of steps taken and the CPU time consumed per step.

The LM algorithm is useful for finding the best-fitting parameters of a nonlinear model to a set of data from a "frequentist" perspective. A Bayesian approach can provide for more robust estimates of parameter uncertainties, especially when those uncertainties are large. One Bayesian method of

\footnotetext{
4 IDL is a commercial programming language and environment by ITT Visual Information Solutions. http://www.ittvis.com/idl/

5 Available at http://purl.com/net/mpfit. MPFIT is a port of MINIPACK-1 from FORTRAN, and is also available in C and Python.
} 
exploring complex or high-dimensional spaces is the Markov chain Monte Carlo method (MCMC; Metropolis et al. 1953; Hastings 1970), which has been productively employed in the context of orbital fitting (e.g., Ford 2004; Driscoll \& Fischer 2005; Balan \& Lahav 2008).

Future space missions, such as NASA's Space Interferometry Mission (SIM Lite), will obtain $\mu$ as astrometry of nearby stars, referenced to an inertial astrometric grid. These measurements will be sufficiently precise to detect Earth-mass planets with orbital periods shorter than the mission lifetime. Since multiple-planet systems are common, ${ }^{6}$ interpreting these data in conjunction with precise $\mathrm{RV}$ data will require algorithms that can efficiently and robustly search the large nonlinear parameter space of multiple-planet systems.

In this paper, we describe the method of efficiently fitting multi-Keplerian models to such high precision RV and astrometric data separating the parameters into linear and nonlinear components in the context of an LM algorithm, and provide the explicit derivatives used in such a fit. With modifications, the principles here can also be applied to MCMC methods, as well (e.g., Catanzarite et al. 2009; Bakos et al. 2009).

\subsection{Plan}

We begin with an elementary exposition to familiarize the reader with our notation (detailed in Table 1) and provide context for the later discussion. In Section 2, we explicate the method of exploiting linear parameters in the Kepler problem using the example of an RV time series. The calculation of explicit derivatives in Section 2.4 is general to any application of the LM algorithm where the model contains both linear and nonlinear parameters. We apply this method to the problem of astrometric data in Section 3 and specifically to the problem of combined astrometric and RV data in Section 3.3. We discuss nonlinear terms relevant to $\mu$ as astrometric work and how to accommodate them in Section 4. In Section 5, we quantify the improvement in speed and convergence from exploiting linear parameters and from the use of explicit derivatives in the LM algorithm.

\section{AN EXAMPLE: RADIAL VELOCITIES}

\subsection{Costs and Benefits of Exploiting Linear Parameters}

When fitting RV data, there are $5 n+1$ Keplerian parameters to be fit, where $n$ is the number of planets ${ }^{7} P$, the period of the planet's orbit; $K$, the semiamplitude of the RV signal; $e$, the eccentricity of the orbit; $\omega$, the argument of periastron; $t_{\mathrm{p}}$, a date of periastron passage; and $\gamma$, the apparent RV of the center of mass of the system. ${ }^{8}$

Exploiting linear parameters, as described below, reduces the search space to $3 n$ dimensions (corresponding to $P$, $e$, and $t_{\mathrm{p}}$ for each planet) and combines the other orbital elements into a set of linear parameters, which can be solved analytically (and therefore quickly and exactly) at each step in the search. The exact, analytic solution of these linear parameters greatly increases the speed and stability of search algorithms, but at a

\footnotetext{
6 At least $1 / 4$ of the known planetary systems show evidence of multiple companions (Wright et al. 2007).

7 Occasionally, an additional "trend" parameter is used to fit out long-term RV trends caused by massive, long-period companions. The other two elements, $i$, the inclination, and $\Omega$, the position angle of the ascending node, can only be determined astrometrically.

8 In practice, $\gamma$ is degenerate with an arbitrary instrument-dependent RV offset.
}

cost: the nonlinear parameters cannot be varied independently of the linear parameters.

Further reduction in the number of nonlinear parameters per planet is certainly possible. By exploiting an epicyclic or harmonic series expansion one can reduce the problem to only one nonlinear parameter per planet, $P_{j}$. Cumming et al. (2003) and Ford (2008) analyze the RV problem in the case of a circular orbit, and discuss the relationship between periodograms and Bayesian approaches to orbit fitting. Konacki \& Maciejewski (1999) pursued a method analogous to ours in their approach to RV curve fitting, and Konacki et al. (2002) did the same for astrometry. Such approximations offer a different set of costs and benefits to the one presented here. For instance, when $K$ and $e$ are both large, it may require a large number of terms to adequately describe a set of RV data. We may pursue such an approach in a future version of our code.

The linear parameters in our treatment are not coefficients in a series expansion; rather, we recast the problem, separating linear parameters, which can be solved for exactly with linear algebra, from nonlinear parameters, which must be solved for algorithmically (using LM). As described in Section 2.2, the linear parameters are algebraic combinations of $K, \omega, \gamma$, and an optional trend parameter, while $P, t_{\mathrm{P}}$, and $e$ are nonlinear parameters.

Two complications are introduced by exploiting linear parameters. First, because the linear parameters are computed analytically, and not algorithmically, their errors and covariances with the nonlinear parameters are not computed automatically in the procedure outlined here. (They also cannot be held fixed in the fit, although the issue of fixing the trend parameter can be finessed-see Section 2.6). The second complication is that computation of the explicit derivatives for the LM algorithm is not straightforward: in the context of the algorithm, the Keplerian model is not a simple function of the $3 n$ nonlinear parameters being fit, but also depends on the data. For example, increasing the nonlinear parameter $e$ slightly not only changes the model because it is more eccentric, but also because at this new value of $e$ the linear parameters have different values. These complications can be avoided if one uses the linear basis of the orbits presented here in an ordinary, nonlinear $5 n+1$ parameter fit, but the convergence and speed-up benefits will not be as great.

The sections below describe a general method of calculating an $n$-planet Keplerian RV model given values for the $3 n$ nonlinear parameters, and of calculating the derivatives of that model with respect to those parameters. These equations can be used in a user-defined function for input to an LM minimization code, or a brute-force $\chi^{2}$ map.

\subsection{Linear Parameters in the Radial Velocity Problem}

We wish to find the parameters of the $n$-planet model

$$
u(t)=\sum_{j=1}^{n}\left[K_{j}\left(\cos \left(\omega_{j}+f_{j}(t)\right)+e_{j} \cos \omega_{j}\right)\right]+\gamma+d \cdot\left(t-t_{0}\right)
$$

which best fits the set of observed radial velocities $\vec{v}$, measured at times $\vec{t}$, with uncertainties $\vec{\sigma}$ in a least-squares sense. Here, $K_{j}$ and $\omega_{j}$ are the usual Keplerian parameters for planet $j, \gamma$ is the time-independent velocity offset, $d$ is the trend parameter, $f_{j}(t)$ is the true anomaly of planet $j$ at time $t$, and $t_{0}$ is a conveniently chosen epoch of the observations. The true anomaly is defined implicitly in terms of the other three Keplerian parameters 
Table 1

Variables and Symbols Used in This Manuscript

\begin{tabular}{|c|c|c|}
\hline Symbol & Meaning & Example Equation \\
\hline & A tilde indicates a first approximation as opposed to a fitted parameter & 93 \\
\hline$A, B, C, F, G, H$ & Thiele-Innes constants & 41 \\
\hline$a$ & Astrometric semimajor axis of a star's orbit in units of arc & 55 \\
\hline$\alpha$ & Nominal right ascension of a system at the epoch of observations, $t_{0}$ & \\
\hline$\hat{\alpha}$ & Constant unit east vector & 89 \\
\hline$\vec{\beta}$ & Vector of linear parameters & 12 \\
\hline$c$ & Linear parameter in RV fitting corresponding to a component of a planet's RV signature & 6 \\
\hline$d$ & Linear parameter in RV fitting corresponding to an RV trend & 6 \\
\hline$D$ & Distance to a system & 93 \\
\hline$\Delta \alpha \cos \delta, \Delta \delta$ & Measured astrometric displacements in units of arc in the $\hat{\alpha}$ and $\hat{\delta}$ directions & 49 and 50 \\
\hline$\delta$ & Nominal declination of a system at the epoch of observations, $t_{0}$ & \\
\hline$\hat{\delta}$ & Constant unit north vector & 90 \\
\hline$\delta_{k l}$ & Kronecker delta & 17 \\
\hline$\gamma$ & Constant (and often instrument dependent) offset in a set of RV data & 1 \\
\hline$E$ & Eccentric anomaly of a planet (a function of time) & 2 \\
\hline$e$ & Eccentricity of a planet & 1 \\
\hline $\mathbf{F}$ & Matrix defined such that the model $\vec{u}=\vec{\beta} \mathbf{F}$ & 15 and 58 \\
\hline$f$ & True anomaly of a planet (a function of time) & 2 \\
\hline$\theta$ & Position angle of astrometric displacement such that $\theta=0$ refers to $\Delta \delta$ & 42 \\
\hline$h$ & Linear parameter in RV fitting corresponding to a component of a planet's RV signature & 5 \\
\hline$i$ & Inclination of a planet's orbit with respect to the sky & 41 \\
\hline j & Subscript indicating a quantity corresponds to the $j$ th planet & 1 \\
\hline$K$ & RV semiamplitude of a star's orbit due to planet & 1 \\
\hline$k$ & Subscript indicating a quantity corresponds to the $k$ th observation & 14 \\
\hline$\lambda$ & A combination of nonlinear orbital parameters & 72 \\
\hline$M$ & Mean anomaly & 3 \\
\hline$m_{*}$ & Mass of the primary component of a binary or planetary system & 55 \\
\hline$m$ & Mass of a smaller component of a binary or planetary system & 55 \\
\hline$\mu, \mu_{\alpha}, \mu_{\delta}$ & Proper motion. The total proper motion is given by $\mu^{2}=\mu_{\alpha}^{2}+\mu_{\delta}^{2}$ & 58 \\
\hline$n$ & Number of planets in a system & 1 \\
\hline$N$ & Number of observations being fit & 15 \\
\hline$\chi^{2}$ & The statistic & 14 \\
\hline$P$ & Period of a planet & 3 \\
\hline$\hat{p}$ & Unit nominal position vector of a star in barycentric equatorial coordinates & 88 \\
\hline$\hat{p}^{\prime}$ & Unit displaced position vector of a star in barycentric equatorial coordinates & 91 and 92 \\
\hline$\Pi_{\alpha}, \Pi_{\delta}$ & Functions (of time and $\vec{r}$ ) describing unit parallactic motion & 53 and 54 \\
\hline$\pi$ & The mathematical constant & \\
\hline$\varpi$ & Parallax of a system & 55 and 58 \\
\hline$R_{x}(\omega), R_{z}(\omega)$ & The three-dimensional rotation matrix about the $x$ - or $z$-axis. & 42 \\
\hline$\vec{r}, r_{x}, r_{y}, r_{z}$ & Observatory position in barycentric equatorial coordinates (a function of time) & 53 and 54 \\
\hline$\rho_{\theta}$ & Measured astrometric displacement in the direction of position angle $\theta$ & 65 \\
\hline$s$ & Scale of random deviation of initial guesses from nominal in units of $\sigma_{x}$ & Section 5.3 \\
\hline$S, T$ & Astrometric terms in the $\mathbf{F}$ matrix & 67 \\
\hline$\sigma$ & Measurement uncertainty & 14 and 17 \\
\hline$\sigma_{x}$ & Uncertainty in an orbital parameter, $x$ & 14 and 17 \\
\hline$t$ & Time of a RV observation & 1 \\
\hline$t_{0}$ & Fiducial time at the epoch of the observations & 93 \\
\hline$t_{\mathrm{p}}$ & Time of periastron passage of a planet & 3 \\
\hline$\tau$ & Time of an astrometric observation & 56,58 \\
\hline$u$ & Model values, such as velocities or astrometric displacements & 1 and 16 \\
\hline$v$ & Measured radial velocities & 14 \\
\hline$v_{0}$ & Linear parameter corresponding to a constant offset in a set of RV data & 7 \\
\hline$v_{r}$ & RV of a system's barycenter with respect to solar system barycenter & 85 \\
\hline W & Diagonal matrix containing weights of measured data & 17 \\
\hline$X, Y$ & Elliptical rectangular coordinates of a planet (functions of time) & 51 and 52 \\
\hline$x$ & As a variable, can stand for any parameter, such as $P_{j}, t_{\mathrm{p}, j}$, or $e_{j}$ & 21 \\
\hline$\Omega$ & Position angle of the ascending (approaching) node of a planet & 41 \\
\hline$\omega$ & Argument of periastron of a planet's orbit & 1 \\
\hline$\omega_{*}$ & Argument of periastron of a star's orbit due to a planet, $\omega=\omega_{*}+\pi$ & 41 \\
\hline
\end{tabular}


$\left(P_{j}, t_{\mathrm{p}, j}\right.$, and $\left.e_{j}\right)$ through the relations

$$
\begin{gathered}
\tan \frac{f_{j}(t)}{2}=\sqrt{\frac{1+e_{j}}{1-e_{j}}} \tan \frac{E_{j}(t)}{2} \\
E_{j}(t)-e_{j} \sin E_{j}(t)=\frac{2 \pi\left(t-t_{\mathrm{p}, j}\right)}{P_{j}}=M_{j}(t) .
\end{gathered}
$$

In Equation (3) (known as Kepler's Equation), $E_{j}$ is called the eccentric anomaly of planet $j$, and $M$ is known as the mean anomaly. ${ }^{9}$

We identify the linear parameters by rewriting Equation (1) as

$$
u(t)=\sum_{j=1}^{n}\left[h_{j} \cos f_{j}(t)+c_{j} \sin f_{j}(t)\right]+v_{0}+d \cdot\left(t-t_{0}\right),
$$

where

$$
\begin{gathered}
h_{j}=K_{j} \cos \omega_{j}, \\
c_{j}=-K_{j} \sin \omega_{j},
\end{gathered}
$$

and

$$
v_{0}=\gamma+\sum_{j=1}^{n} K_{j} e_{j} \cos \omega_{j}
$$

These linear parameters can be converted back to Keplerian orbital elements through the relations

$$
\begin{gathered}
K_{j}=\sqrt{h_{j}^{2}+c_{j}^{2}} \\
\tan \omega_{j}=\frac{-c_{j}}{h_{j}}
\end{gathered}
$$

(where $\omega_{j}$ is chosen so that $\sin \omega_{j}$ has the sign of the numerator) and

$$
\gamma=v_{0}-\sum_{j=1}^{n} K_{j} e_{j} \cos \omega_{j}
$$

The masses of the orbiting planets can be inferred from their corresponding semiamplitudes $K_{j}$, defined for a single-planet system with planet mass $m$ and stellar mass $m_{*}$ as

$$
K^{3}=\frac{2 \pi G}{P\left(1-e^{2}\right)^{\frac{3}{2}}}\left(\frac{m^{3} \sin ^{3} i}{\left(m_{*}+m\right)^{2}}\right),
$$

where $G$ is Newton's gravitational constant. The fraction in parentheses is known as the mass function of the system. The problem can now be divided into two parts: an algorithmic search through parameter space for the best-fit nonlinear parameters $P_{j}, e_{j}$, and $t_{\mathrm{p}, j}$ with a computer routine such as an LM or an MCMC code, and at each step in that search an analytic solution for the linear parameters that fit best there.

\footnotetext{
$9 \quad$ Instead of $t_{\mathrm{p}}$, many authors (especially dynamicists) prefer to parameterize
} orbits in terms of the mean longitude at epoch, defined as $M\left(t_{0}\right)+\Omega+\omega$.

\subsection{Solving for the Linear Parameters}

Given a set of values for the nonlinear parameters, we can fit for the linear parameters in Equation (4) through $\chi^{2}$ minimization. We denote the row vector of linear parameters

$$
\vec{\beta}=\left\{h_{1}, c_{1}, h_{2}, c_{2} \ldots h_{n}, c_{n}, v_{0}, d\right\}
$$

We define $\chi^{2}$ the usual way:

$$
\chi^{2}=\sum_{k=1}^{N} \frac{\left(v_{k}-u\left(t_{k}\right)\right)^{2}}{\sigma_{k}^{2}}
$$

and minimize it with respect to each of the linear parameters in $\vec{\beta}$ simultaneously:

$$
\frac{\partial \chi^{2}}{\partial \beta_{l}}=-\left.2 \sum_{k=1}^{N} \frac{v_{k}-u\left(t_{k}\right)}{\sigma_{k}^{2}} \frac{\partial u}{\partial \beta_{l}}\right|_{t=t_{k}}=0
$$

We can express this more compactly by invoking matrix algebra. For the problem of Keplerian orbits, we define the matrix $\mathbf{F}$ as

$$
\mathbf{F}=\left[\begin{array}{ccc}
\cos f_{1,1} & \cos f_{1,2} & \ldots \\
\sin f_{1,1} & \sin f_{1,2} & \ldots \\
\cos f_{2,1} & \cos f_{2,2} & \ldots \\
\sin f_{2,1} & \sin f_{2,2} & \ldots \\
\vdots & \vdots & \\
\cos f_{n, 1} & \cos f_{n, 2} & \ldots \\
\sin f_{n, 1} & \sin f_{n, 2} & \ldots \\
1 & 1 & \ldots \\
t_{1}-t_{0} & t_{2}-t_{0} & \ldots
\end{array}\right]
$$

where $f_{j, k} \equiv f_{j}\left(t_{k}\right)$. This allows us to write the model velocities at times $\vec{t}$ (Equation (1)) as

$$
\vec{u}=\vec{\beta} \mathbf{F}
$$

We also define the diagonal weight matrix $\mathbf{W}$ such that

$$
W_{k l}=\delta_{k l} / \sigma_{k}^{2}
$$

where we have used the Kronecker delta symbol. We can then write the system of equations in Equation (14) as

$$
\frac{\partial \chi^{2}}{\partial \vec{\beta}}=-2(\vec{v}-\vec{\beta} \mathbf{F}) \mathbf{W} \mathbf{F}^{\mathrm{T}}=\overrightarrow{0}
$$

Solving for $\vec{\beta}$ we have

$$
\vec{\beta}=\vec{v} \mathbf{W F}^{\mathrm{T}} \boldsymbol{\epsilon},
$$

where we have denoted the error matrix (also called the variancecovariance matrix)

$$
\left.\boldsymbol{\epsilon}=(\mathbf{F W F})^{\mathrm{T}}\right)^{-1}
$$

Equation (19) represents the general solution to the linear least-squares problem for an appropriately defined F. In the context of Keplerian fits, given a set of nonlinear parameters $P, e$, and $t_{\mathrm{p}}$ for each planet, the remaining Keplerian orbital elements can be found from $\vec{\beta}$ using Equations (8)-(10). 


\subsection{Calculation of Explicit (Analytic) Derivatives for Use in the LM Algorithm}

The derivative of the model velocities $\vec{u}$ with respect to any nonlinear parameter can be found from Equation (16):

$$
\frac{d \vec{u}}{d x}=\frac{d \vec{\beta}}{d x} \mathbf{F}+\vec{\beta} \frac{d \mathbf{F}}{d x},
$$

where $x$ stands for any of the nonlinear parameters (here, $P_{j}, e_{j}$, or $t_{\mathrm{p}, j}$ ).

From Equation (19) we have

$$
\frac{d \vec{\beta}}{d x}=\vec{v} \mathbf{W}\left(\frac{d \mathbf{F}^{\mathrm{T}}}{d x} \boldsymbol{\epsilon}+\mathbf{F}^{\mathrm{T}} \frac{d \boldsymbol{\epsilon}}{d x}\right) .
$$

From the definition of a matrix inverse we can express the last derivative as

$$
\frac{d \boldsymbol{\epsilon}}{d x}=-\boldsymbol{\epsilon}\left(\frac{d}{d x} \boldsymbol{\epsilon}^{-1}\right) \boldsymbol{\epsilon} .
$$

Using Equation (20) we then have

$$
\begin{gathered}
\frac{d \boldsymbol{\epsilon}}{d x}=-\boldsymbol{\epsilon} \frac{d}{d x}\left(\mathbf{F} \mathbf{W} \mathbf{F}^{\mathrm{T}}\right) \boldsymbol{\epsilon} \\
=-\boldsymbol{\epsilon}\left(\left(\frac{d \mathbf{F}}{d x} \mathbf{W} \mathbf{F}^{\mathrm{T}}\right)+\left(\frac{d \mathbf{F}}{d x} \mathbf{W F}^{\mathrm{T}}\right)^{\mathrm{T}}\right) \boldsymbol{\epsilon} .
\end{gathered}
$$

Equations (16)-(24) are not specific to the RV Kepler problem, but are a general method of calculating explicit derivatives in a model with both linear and nonlinear parameters, and so can be applied to any analogous problem. For instance, the problem of fitting an orbit using astrometric data also has linear parameters as we show in Section 3.

\subsection{Explicit Derivatives for the Radial Velocity Model}

For the case of a Keplerian RV model, from Equation (15) we have

$$
\frac{d \mathbf{F}}{d x}=\left[\begin{array}{ccc}
-\sin f_{1,1} f_{1,1}^{\prime} & -\sin f_{1,2} f_{1,2}^{\prime} & \cdots \\
\cos f_{1,1} f_{1,1}^{\prime} & \cos f_{1,2} f_{1,2}^{\prime} & \cdots \\
-\sin f_{2,1} f_{2,1}^{\prime} & -\sin f_{2,2} f_{2,2}^{\prime} & \cdots \\
\cos f_{2,1} f_{2,1}^{\prime} & \cos f_{2,2} f_{2,2}^{\prime} & \cdots \\
\vdots & \vdots & \\
-\sin f_{n, 1} f_{n, 1}^{\prime} & -\sin f_{n, 2} f_{n, 2}^{\prime} & \cdots \\
\cos f_{n, 1} f_{n, 1}^{\prime} & \cos f_{n, 2} f_{n, 2}^{\prime} & \cdots \\
0 & 0 & \cdots \\
0 & 0 & \cdots
\end{array}\right],
$$

where $f_{j, k}^{\prime} \equiv d f_{j, k} / d x$. Note that since $f_{j, k}^{\prime}$ refers to the true anomaly of planet $j$, it vanishes when $x$ refers to a parameter of a different planet (e.g., $d f_{j, k} / d P_{l}=0$ when $j \neq l$ ). This means that the matrix $d \mathbf{F} / d x$ has only two nonzero rows. We can therefore suppress subscripts below for clarity.

We can calculate the nonzero derivatives as

$$
\frac{d f}{d x}=\frac{\partial f}{\partial x}+\frac{\partial f}{\partial E} \frac{d E}{d x},
$$

where, from Kepler's equation (Equation (3)), we have

$$
\frac{d E}{d P}=\frac{-2 \pi\left(t-t_{\mathrm{p}}\right) / P^{2}}{1-e \cos E}
$$

$$
\begin{aligned}
& \frac{d E}{d t_{\mathrm{p}}}=\frac{-2 \pi / P}{1-e \cos E} \\
& \frac{d E}{d e}=\frac{\sin E}{1-e \cos E}
\end{aligned}
$$

and from Equation (2) we have

$$
\frac{\partial f}{\partial E}=\sqrt{\frac{1+e}{1-e}} \frac{1+\cos f}{1+\cos E} .
$$

From Equation (2) we can also write

$$
\frac{\partial f}{\partial e}=\frac{2 \tan (E / 2) \cos ^{2}(f / 2)}{(1-e) \sqrt{1-e^{2}}},
$$

but it is more computationally convenient to note that this happens to simplify to

$$
\frac{\partial f}{\partial e}=\frac{\partial f}{\partial E} \frac{\sin E}{1-e^{2}} .
$$

Finally,

$$
\frac{\partial f}{\partial P}=\frac{\partial f}{\partial t_{\mathrm{p}}}=0 .
$$

Equations (22)-(34) can be used to calculate the terms in Equation (21), yielding the explicit derivatives used by LM method fitting routines, such as MPFIT.

\subsection{Variations on the $n$-Planet RV Model}

In the context of the Kepler problem, the above equations include a parameter for a linear trend in the data. In practice, fitting for such a trend will only occasionally be necessary. When not needed the $d$ parameter in $\vec{\beta}$ and the bottom rows of the $\mathbf{F}$ and $d \mathbf{F} / d x$ matrices can simply be left out of the calculations. Similarly, the trend parameter can effectively be held fixed at a nonzero value by simply subtracting the desired value from the data before fitting.

These matrices can also be easily extended to handle the case of combining data from multiple telescopes between which there exist RV offsets. This is accomplished by extending the data vectors $\vec{v}, \vec{t}$, and $\vec{\sigma}$ to include data from all telescopes, and extending the vector $\vec{\beta}$ to include a separate offset parameter for each telescope after the first. The corresponding rows of $\mathbf{F}$ must then be filled with $1 \mathrm{~s}$ in those columns corresponding to data from the appropriate telescope, and 0s elsewhere. Naturally, in $d \mathbf{F} / d x$ the elements of these rows are all 0.

\section{APPLICATIONS TO ASTROMETRY}

\subsection{Astrometry Alone}

The above method can also be extended to the problem of fitting for the Keplerian elements of an orbit from astrometric data. Here, we present a simplified model of $\mu$ as astrometric data of the sort that might be provided by a space observatory such as NASA's SIM Lite. We anticipate modifying our algorithms for a more realistic model of interferometric data and its noise sources (Sozzetti 2005; Eriksson \& Lindegren 2007; Catanzarite et al. 2008) in the near future.

The linear basis for astrometric coordinates are the ThieleInnes constants (e.g., Binnendijk 1960), and are well documented as useful tools for astrometric curve-fitting (e.g., Casertano et al. 2008). The astrometric perturbations caused 
by an orbiting companion can be described in terms of six astrometric orbital elements: in addition to $e$ and $t_{\mathrm{p}}$, we have $a$, the semimajor axis of the star's apparent orbit on the sky in units of arc; $\Omega$, the longitude of the ascending (approaching) node (measured as a position angle on the sky); $i$, the inclination of the orbit on the sky (such that $i=0$ corresponds to a face-on, clockwise orbit); and $\omega_{*}$, the longitude of periastron of the star's orbit. ${ }^{10}$

The Thiele-Innes constants are defined in terms of four of the astrometric elements of the star's orbit about the secondary:

$$
\begin{gathered}
A=a\left(\cos \Omega \cos \omega_{*}-\sin \Omega \sin \omega_{*} \cos i\right) \\
B=a\left(\sin \Omega \cos \omega_{*}+\cos \Omega \sin \omega_{*} \cos i\right) \\
F=a\left(-\cos \Omega \sin \omega_{*}-\sin \Omega \cos \omega_{*} \cos i\right) \\
G=a\left(-\sin \Omega \sin \omega_{*}+\cos \Omega \cos \omega_{*} \cos i\right) \\
C=a \sin \omega_{*} \sin i \\
H=a \cos \omega_{*} \sin i .
\end{gathered}
$$

These constants can be quickly computed using rotation matrices:

$$
\begin{aligned}
& {\left[\begin{array}{ccc}
A & B & C \\
F & G & H \\
a \sin i \sin \Omega & -a \sin i \cos \Omega & a \cos i
\end{array}\right]} \\
& =a R_{z}\left(\omega_{*}\right) R_{x}(i) R_{z}(\Omega),
\end{aligned}
$$

where $R$ is the three-dimensional rotation matrix

$$
R_{z}(\Omega)=\left[\begin{array}{ccc}
\cos \Omega & \sin \Omega & 0 \\
-\sin \Omega & \cos \Omega & 0 \\
0 & 0 & 1
\end{array}\right]
$$

and $\omega_{*}$ is the argument of periastron of the orbit of the star.

We can transform the Thiele-Innes constants back to Keplerian orbital elements of the planet with the relations:

$$
\begin{gathered}
\tan \left(\omega_{*}+\Omega\right)=\frac{B-F}{A+G} \\
\tan \left(\omega_{*}-\Omega\right)=\frac{-(B+F)}{A-G} \\
\tan ^{2}\left(\frac{i}{2}\right)=\frac{(A-G) \cos \left(\omega_{*}+\Omega\right)}{(A+G) \cos \left(\omega_{*}-\Omega\right)} \\
a=\quad\left(A \cos \omega_{*}-F \sin \omega_{*}\right) \cos \Omega \\
-\left(A \sin \omega_{*}+F \cos \omega_{*}\right) \sin \Omega \sec \mathrm{i}
\end{gathered}
$$

and

$$
\omega=\omega_{*}+\pi,
$$

where the quadrants of $\omega_{*}-\Omega$ and $\omega_{*}+\Omega$ are determined by the signs of the numerators in Equations (43) and (44). These

\footnotetext{
10 The orbital parameters of the star and those of the unseen companion are all identical except $a$, which differs by a factor of $m / m_{*}$, and $\omega$, which differs by $\pi$.
}

relations leave a $\pm \pi$ ambiguity in $\omega_{*}, \omega$, and $\Omega$ that can only be resolved by radial velocities, without which convention dictates that we choose the solution with $\Omega<\pi$.

The Thiele-Innes constants $C$ and $H$ are closely related to the $c$ and $h$ constants of Equations (5) and (6). The set of six constants have the identity

$$
a^{2}=A^{2}+B^{2}+C^{2}=F^{2}+G^{2}+H^{2} .
$$

In astrometry there are five parameters that describe a star's motion in the absence of orbiting companions: $\Delta \alpha_{0} \cos \delta$ and $\Delta \delta_{0}$, the difference between the true and nominal position of the system at $t_{0} ; \mu_{\alpha}$ and $\mu_{\delta}$, the proper motions in the R.A. and decl. directions; and $\varpi$, the parallax of the system. Our model for the astrometric displacement of a star due to parallax, proper motion, and a system of unseen planets in terms of the Thiele-Innes constants at times $\vec{\tau}$ is

$$
\begin{gathered}
\Delta \delta_{k}=\sum_{j=1}^{n}\left[A_{j} X_{j, k}+F_{j} Y_{j, k}\right] \\
+\Delta \delta_{0}+\varpi \prod_{\delta, k}+\mu_{\delta}\left(\tau_{k}-t_{0}\right) \\
\Delta \alpha_{k} \cos \delta=\sum_{j=1}^{n}\left[B_{j} X_{j, k}+G_{j} Y_{j, k}\right] \\
+\Delta \alpha_{0} \cos \delta+\varpi \prod_{\alpha, k}+\mu_{\alpha}\left(\tau_{k}-t_{0}\right),
\end{gathered}
$$

where $X$ and $Y$ are the so-called elliptical rectangular coordinates, defined as

$$
\begin{gathered}
X_{j, k}=\cos E_{j}\left(\tau_{k}\right)-e_{j} \\
Y_{j, k}=\sqrt{1-e_{j}^{2}} \sin E_{j}\left(\tau_{k}\right),
\end{gathered}
$$

where $E$ is the eccentric anomaly and the quantities $\Pi_{\alpha, k}$ and $\Pi_{\delta, k}$ refer to the astrometric displacements due to parallax in the $\alpha$ and $\delta$ directions ${ }^{11}$ during observation $k$, which are given by Seidelman (1992).

$$
\Pi_{\alpha, k}=r_{x}\left(\tau_{k}\right) \sin \alpha-r_{y}\left(\tau_{k}\right) \cos \alpha
$$

$$
\Pi_{\delta, k}=\left(r_{x}\left(\tau_{k}\right) \cos \alpha+r_{y}\left(\tau_{k}\right) \sin \alpha\right) \sin \delta-r_{z}\left(\tau_{k}\right) \cos \delta .
$$

Here, $\left(r_{x}, r_{y}, r_{z}\right)$ represent the Cartesian components in equatorial coordinates of the position of the observatory, $\vec{r}$, at time $\tau$ with respect to the solar system barycenter (in units of AU when $\varpi$ is in arcsec). These values for the Earth are available from the NASA Jet Propulsion Laboratory Solar System ephimerides, ${ }^{12}$ but for $\mu$ as and spaceborne work the precise position of the observatory itself is required.

Note that since $a$ is the apparent semimajor axis of the star's orbit in units of arc, its relationship to the mass of the secondary depends on the method of astrometry used. For astrometric perturbations due to an unseen planet (that is, absolute astrometric displacements with respect to the sidereal frame, as measured by SIM Lite), we have from Kepler's Third Law

$$
a^{3}=\frac{\varpi^{3} m^{3}}{\left(m_{*}+m\right)^{2}} P^{2},
$$

where $a$ is measured in arcseconds when $P$ is measured in years, $\varpi$ is the parallax in arcseconds, and $m$ is the mass of the unseen companion and $m_{*}$ is the mass of the star in solar masses. ${ }^{13}$

\footnotetext{
11 In this paper, the bare symbols $\alpha$ and $\delta$ will always refer to the nominal right ascension and declination of a star at $t_{0}$, absent the effects of parallax and astrometric displacement from companions.

12 http://ssd.jpl.nasa.gov

13 In the case where the binary orbit is measured as a separation and position angle of one star with respect to another (i.e., relative astrometry) the measured separation is given by $a^{3}=\varpi^{3}\left(m_{*}+m\right) P^{2}$. The application of the techniques here for multiple-planet systems with relative astrometry is not straightforward, and is beyond the scope of this manuscript
} 
We can now extend Equation (16) to the case of twodimensional data by defining our vector of $N$ measurements taken at times $\vec{\tau}$ :

$$
\left[\Delta \delta_{1}, \Delta \delta_{2} \ldots \Delta \delta_{N}, \Delta \alpha_{1} \cos \delta, \Delta \alpha_{2} \cos \delta \ldots \Delta \alpha_{N} \cos \delta\right]
$$

and our model with linear parameters for $n$ planets:

$$
\begin{aligned}
\vec{\beta}= & {\left[A_{1}, B_{1}, F_{1}, G_{1} \ldots A_{n}, B_{n}, F_{n}, G_{n},\right.} \\
& \left.\Delta \delta_{0}, \Delta \alpha_{0} \cos \delta, \mu_{\delta}, \mu_{\alpha}, \varpi\right]
\end{aligned}
$$

\begin{tabular}{|c|c|c|c|c|c|c|c|}
\hline$X_{1,1}$ & $X_{1,2}$ & $\ldots$ & $X_{1, N}$ & 0 & 0 & $\ldots$ & 0 \\
\hline 0 & 0 & $\ldots$ & 0 & $X_{1,1}$ & $X_{1,2}$ & $\ldots$ & $X_{1, N}$ \\
\hline$Y_{1,1}$ & $Y_{1,2}$ & $\ldots$ & $Y_{1, N}$ & 0 & 0 & $\ldots$ & 0 \\
\hline 0 & 0 & $\ldots$ & 0 & $Y_{1,1}$ & $Y_{1,2}$ & $\ldots$ & $Y_{1, N}$ \\
\hline$X_{2,1}$ & $X_{2,2}$ & $\ldots$ & $X_{2, N}$ & 0 & 0 & $\ldots$ & 0 \\
\hline 0 & 0 & $\ldots$ & 0 & $X_{2,1}$ & $X_{2,2}$ & $\ldots$ & $X_{2, N}$ \\
\hline$Y_{2,1}$ & $Y_{2,2}$ & $\ldots$ & $Y_{2, N}$ & 0 & 0 & $\ldots$ & 0 \\
\hline 0 & 0 & $\ldots$ & 0 & $Y_{2,1}$ & $Y_{2,2}$ & $\ldots$ & $Y_{2, N}$ \\
\hline$\vdots$ & $\vdots$ & & $\vdots$ & $\vdots$ & $\vdots$ & & $\vdots$ \\
\hline$X_{n, 1}$ & $X_{n, 2}$ & $\ldots$ & $X_{n, N}$ & 0 & 0 & $\ldots$ & 0 \\
\hline 0 & 0 & $\ldots$ & 0 & $X_{n, 1}$ & $X_{n, 2}$ & $\ldots$ & $X_{n, N}$ \\
\hline$Y_{n, 1}$ & $Y_{n, 2}$ & $\ldots$ & $Y_{n, N}$ & 0 & 0 & $\ldots$ & 0 \\
\hline 0 & 0 & $\ldots$ & 0 & $Y_{n, 1}$ & $Y_{n, 2}$ & $\ldots$ & $Y_{n, N}$ \\
\hline 1 & 1 & $\ldots$ & 1 & 0 & 0 & $\ldots$ & 0 \\
\hline 0 & 0 & $\ldots$ & 0 & 1 & 1 & $\ldots$ & 1 \\
\hline$\tau_{1}-t_{0}$ & $\tau_{2}-t_{0}$ & $\ldots$ & $\tau_{N}-t_{0}$ & 0 & 0 & $\ldots$ & 0 \\
\hline 0 & 0 & $\ldots$ & 0 & $\tau_{1}-t_{0}$ & $\tau_{2}-t_{0}$ & $\ldots$ & $\tau_{N}-t_{0}$ \\
\hline$\Pi_{\delta, 1}$ & $\Pi_{\delta, 2}$ & $\ldots$ & $\Pi_{\delta, N}$ & $\Pi_{\alpha, 1}$ & $\Pi_{\alpha, 2}$ & $\ldots$ & $\Pi_{\alpha, N}$ \\
\hline
\end{tabular}

and matrix $\mathbf{F}=$

The nonzero components of $d \mathbf{F} / d x$ can be calculated from

$$
\begin{gathered}
\frac{d X}{d P}=-\frac{d E}{d P} \sin E \\
\frac{d X}{d t_{\mathrm{p}}}=-\frac{d E}{d t_{\mathrm{p}}} \sin E \\
\frac{d X}{d e}=-\frac{d E}{d e} \sin E-1 \\
\frac{d Y}{d P}=\sqrt{1-e^{2}} \cos E \frac{d E}{d P} \\
\frac{d Y}{d t_{\mathrm{p}}}=\sqrt{1-e^{2}} \cos E \frac{d E}{d t_{\mathrm{p}}} \\
\frac{d Y}{d e}=\sqrt{1-e^{2}} \cos E \frac{d E}{d e}-\frac{e \sin E}{\sqrt{1-e^{2}}}
\end{gathered}
$$

and Equations (28)-(30).

\subsection{Astrometry in Arbitrary Coordinates}

Astrometry does not always deliver contemporaneous $(\Delta \alpha \cos \delta, \Delta \delta)$ pairs at a common time $t$. In the general case, a baseline determines the one-dimensional displacement of a star from some reference at an intermediate position angle on the sky (i.e., not necessarily 0 [as in the case for $\Delta \delta$ ] or $\pi / 2$ [as for $\Delta \alpha \cos \delta$ ]). We can combine Equations (49) and (50) to a more general form to accommodate a heterogeneous set of such data:

$$
\begin{aligned}
\rho_{\theta, k}= & \sum_{j=1}^{n}\left[\left(A_{j} X_{j, k}+F_{j} Y_{j, k}\right) \cos \theta_{k}\right. \\
& +\left[\left(B_{j} X_{j, k}+G_{j} Y_{j k}\right) \sin \theta_{k}\right] \\
& +\left(\Delta \delta_{0}+\varpi \Pi_{\delta, k}+\mu_{\delta}\left(\tau_{k}-t_{0}\right)\right) \cos \theta_{k} \\
& +\left(\Delta \alpha_{0} \cos \delta+\varpi \Pi_{\alpha, k}+\mu_{\alpha}\left(\tau_{k}-t_{0}\right)\right) \sin \theta_{k},
\end{aligned}
$$

where $\rho$ is the separation and $\theta$ is the position angle of the measurement.

Interestingly, we could also use the other two Thiele-Innes constants to achieve the same result by defining a new linear parameter scheme where the astrometric displacements are described as

$$
\begin{aligned}
\rho_{\theta, k}= & \sum_{j=1}^{n}\left[H_{j} S_{j, k}+C_{j} T_{j, k}\right] \\
& +\left(\Delta \delta_{0}+\varpi \Pi_{\delta, k}+\mu_{\delta}\left(\tau_{k}-t_{0}\right)\right) \cos \theta_{k} \\
& +\left(\Delta \alpha_{0} \cos \delta+\varpi \Pi_{\alpha, k}+\mu_{\alpha}\left(\tau_{k}-t_{0}\right)\right) \sin \theta_{k},
\end{aligned}
$$

where $S$ and $T$ are defined for the $k$ th measurement and $j$ th planet as

$$
\begin{aligned}
& {\left[\begin{array}{l}
S_{j, k} \\
T_{j, k}
\end{array}\right]=} \\
& {\left[\begin{array}{c}
\cos \left(\Omega_{j}-\theta_{k}\right) \csc i_{j}-\sin \left(\Omega_{j}-\theta_{k}\right) \cot i_{j} \\
-\sin \left(\Omega_{j}-\theta_{k}\right) \cot i_{j}-\cos \left(\Omega_{j}-\theta_{k}\right) \csc i_{j}
\end{array}\right]\left[\begin{array}{c}
X_{j, k} \\
Y_{j, k}
\end{array}\right] .}
\end{aligned}
$$

This scheme uses only two linear parameters, instead of four, per planet, and so will not be as efficient, but it is still useful because it can be easily combined with the RV scheme of Section 2.2. We demonstrate this in Section 3.3 with a procedure that can accommodate any combination of RV and astrometric data.

We can recover the parameters $a$ and $\omega$ from the linear parameters $C$ and $H$ given the nonlinear parameter $i$ by

$$
\begin{gathered}
a_{j}^{2}=\frac{C_{j}^{2}+H_{j}^{2}}{\sin ^{2} i_{j}} \\
\tan \omega_{j}=\frac{-C_{j}}{-H_{j}},
\end{gathered}
$$

where $\omega_{j}$ is chosen so $\sin \omega_{j}$ has the same sign as $-C_{j}$.

\subsection{Combining Astrometry with Radial Velocities}

Combining astrometric data with RV data is not as simple as combining the RV-only and astrometry-only schemes outlined above, because the six linear parameters $(A, B, F, G$, and $c$ and $h$ ) are a combination of only five Keplerian elements ( $K, a, \Omega, \omega$, and $i$ ), and the problem is thus overconstrained. One solution would be to minimize $\chi^{2}$ subject to the appropriate constraints using Lagrange multipliers, but the resulting set of nonlinear equations may not be guaranteed to have a unique solution and would be difficult to solve for the case of an arbitrary number of planets.

Another solution is to adapt the $C$ and $H$ constants to accommodate general astrometric data, and these constants are closely related to the scheme for RV data used in Section 2.2. To do this, we define our vector of measurements to be

$$
\left[\vec{v}, \vec{\rho}_{\vec{\theta}}\right]
$$

where the velocities are taken at times $\vec{t}$ and the astrometry at times $\vec{\tau}$, and define our model, as in Equation (16):

$$
\begin{aligned}
\vec{\beta}= & {\left[H_{1}, C_{1}, H_{2}, C_{2} \ldots H_{n}, C_{n}, v_{0}, d,\right.} \\
& \left.\Delta \delta_{0}, \Delta \alpha_{0} \cos \delta, \mu_{\delta}, \mu_{\alpha}, \varpi\right] .
\end{aligned}
$$

From Equations (5), (6), (41), and (55) we have

$$
h_{j}=-\lambda_{j} H_{j}
$$




$$
c_{j}=\lambda_{j} C_{j},
$$

where we have introduced a $\lambda$, a combination of nonlinear orbital parameters of planet $j$ which has units of velocity and has the value

$$
\lambda_{j}=\frac{2 \pi \mathrm{AU}}{\tilde{\varpi} P_{j} \sqrt{1-e_{j}^{2}}}
$$

when the estimated parallax, $\tilde{\varpi}$, is expressed in arcseconds.

The parameters $H_{j}, C_{j}$, and $v_{0}$ can be transformed into $m_{j}^{3} /\left(m_{*}+m_{j}\right)^{2}, \omega_{j}$, and $\gamma$ with Equations (8)-(11), (72), and (73).

The appearance of $\varpi$ in the definition of $\lambda_{j}$ (and therefore in $C_{j}$ and $H_{j}$ ) indicates a fundamental nonlinearity in the combined RV-astrometry problem: the parallax is not a truly linear parameter. However, because it is nearly linear, if a good estimate of the parallax is available then this can be used in the formula for $\lambda_{j}$ (we indicate the approximate nature of the parallax term with a tilde). Once the parallax is solved for more precisely, the fit can be re-run with an improved estimate of $\varpi$. This procedure should converge very quickly, and we will use it again to deal with other, smaller nonlinear terms in Section 4.

The first columns of the $\mathbf{F}$ matrix, corresponding to the RV measurements, now read

$$
\left[\begin{array}{ccl}
-\cos f_{1,1} \lambda_{1} & -\cos f_{1,2} \lambda_{1} & \cdots \\
\sin f_{1,1} \lambda_{1} & \sin f_{1,2} \lambda_{1} & \cdots \\
-\cos f_{2,1} \lambda_{2} & -\cos f_{2,2} \lambda_{2} & \cdots \\
\sin f_{2,1} \lambda_{2} & \sin f_{2,2} \lambda_{2} & \cdots \\
\vdots & \vdots & \\
-\cos f_{n, 1} \lambda_{n} & -\cos f_{n, 2} \lambda_{n} & \cdots \\
\sin f_{n, 1} \lambda_{n} & \sin f_{n, 2} \lambda_{n} & \cdots \\
1 & 1 & \cdots \\
t_{1}-t_{0} & t_{2}-t_{0} & \cdots \\
0 & 0 & \cdots \\
0 & 0 & \cdots \\
0 & 0 & \cdots \\
0 & 0 & \cdots \\
0 & 0 & \cdots
\end{array}\right.
$$

and the rest of the columns, corresponding to the astrometric measurements, read

$$
\begin{array}{ccc}
S_{1,1} & S_{1,2} & \ldots \\
T_{1,1} & T_{1,2} & \ldots \\
S_{2,1} & S_{2,2} & \ldots \\
T_{2,1} & T_{2,2} & \ldots \\
\vdots & \vdots & \\
S_{n, 1} & S_{n, 2} & \ldots \\
T_{n, 1} & T_{n, 2} & \ldots \\
0 & 0 & \ldots \\
0 & 0 & \ldots \\
\cos \theta_{1} & \cos \theta_{2} & \ldots \\
\sin \theta_{1} & \sin \theta_{2} & \ldots \\
\left.-t_{0}\right) \cos \theta_{1} & \left(\tau_{2}-t_{0}\right) \cos \theta_{2} & \ldots \\
\left.-t_{0}\right) \sin \theta_{1} & \left(\tau_{2}-t_{0}\right) \sin \theta_{2} & \ldots \\
\theta_{1}+\prod_{\alpha, 1} \sin \theta_{1} & \Pi_{\delta, 2} \cos \theta_{2}+\Pi_{\alpha, 2} \sin \theta_{2} & \ldots
\end{array}
$$

For the nonlinear parameters $P_{j}, T_{\mathrm{p}, j}$, and $e_{j}$, the two nonzero rows of $d \mathbf{F} / d x$ can be calculated from

$$
\frac{d}{d x}(\cos f(t) \lambda)=-\sin f(t) f^{\prime}(t) \lambda+\cos f(t) \frac{d \lambda}{d x}
$$

$$
\frac{d}{d x}(-\sin f(t) \lambda)=-\cos f(t) f^{\prime}(t) \lambda-\sin f(t) \frac{d \lambda}{d x},
$$

where

$$
\begin{gathered}
\frac{d \lambda}{d P}=-\frac{\lambda}{P} \\
\frac{d \lambda}{d e}=\frac{e \lambda}{1-e^{2}} \\
\frac{d \lambda}{d \Omega}=\frac{d \lambda}{d i}=\frac{d \lambda}{d t_{\mathrm{p}}}=0
\end{gathered}
$$

and

$$
\begin{aligned}
& {\left[\begin{array}{l}
d S / d x \\
d T / d x
\end{array}\right]=} \\
& {\left[\begin{array}{c}
\cos (\Omega-\theta) \csc i-\sin (\Omega-\theta) \cot i \\
-\sin (\Omega-\theta) \cot i-\cos (\Omega-\theta) \csc i
\end{array}\right]\left[\begin{array}{l}
d X / d x \\
d Y / d x
\end{array}\right]}
\end{aligned}
$$

and Equations (27)-(34), (59)-(64). Now we have introduced two additional nonlinear parameters, $\Omega$ and $i$ :

$$
\begin{aligned}
& {\left[\begin{array}{l}
d S / d \Omega \\
d T / d \Omega
\end{array}\right]=} \\
& {\left[\begin{array}{l}
-\sin (\Omega-\theta) \csc i-\cos (\Omega-\theta) \cot i \\
-\cos (\Omega-\theta) \cot i \\
\sin (\Omega-\theta) \csc i
\end{array}\right]\left[\begin{array}{l}
X \\
Y
\end{array}\right]}
\end{aligned}
$$

and

$$
\begin{aligned}
& {\left[\begin{array}{l}
d S / d i \\
d T / d i
\end{array}\right]=} \\
& {\left[\begin{array}{cc}
-\cos (\Omega-\theta) \csc i \cot i & \sin (\Omega-\theta) \csc ^{2} i \\
\sin (\Omega-\theta) \csc ^{2} i & \cos (\Omega-\theta) \csc i \cot i
\end{array}\right]\left[\begin{array}{l}
X \\
Y
\end{array}\right] .}
\end{aligned}
$$

\section{NONLINEAR TERMS}

\subsection{Sources of Nonlinearity}

There are several small nonlinear terms which are important at the $\mathrm{m} \mathrm{s}^{-1}$ and $\mu$ as level, especially for the nearby and high proper motion stars likely to be observed by SIM Lite.

Secular acceleration. A star with significant proper motion will have the radial component of its space velocity change with position on the sky, resulting in a secular change in the RV up to $\sim 1 \mathrm{~m} \mathrm{~s}^{-1} \mathrm{yr}^{-1}$ for the most extreme cases. Secular acceleration is given, to first order, by

$$
\dot{v}_{r}=D \mu^{2},
$$

where, $v_{r}$ is the bulk RV of the star, ${ }^{14} D$ is the star's distance, ${ }^{15}$ and $\mu$ is the total proper motion in radians per unit time. This

\footnotetext{
14 This quantity $v_{r}$ defines the true RV of the system with respect to the solar system barycenter. It differs from the spectroscopic parameter $\gamma$ in that the latter is often measured with respect to a fiducial frame and can include non-Doppler effects such as instrumental offsets, gravitational redshift, and convective blueshift.

${ }^{15} D$ is distinguished here from the inverse parallax $\varpi^{-1}$ simply for convenience of units.
} 
term will be absorbed into the linear parameter $d$, if present, and so could be ignored.

Parallax changes. The change in parallax of nearby stars due to their RV may be of the order of $0.3 \mu \mathrm{as} \mathrm{yr}^{-1}$. The shape of the parallactic motion is also a function of position on the sky, and thus of the proper motion. These changes can be of the order of $5 \mu$ as $\mathrm{yr}^{-1}$. The RV term is given by

$$
\dot{\varpi}=-\varpi \frac{v_{r}}{D} \text {. }
$$

Proper motion changes. The flip side of secular acceleration is proper motion change due to change in distance. This effect can be of the order of $3 \mu$ as $\mathrm{yr}^{-1}$ and is given by

$$
\dot{\vec{\mu}}=-\vec{\mu} \frac{v_{r}}{D} \text {. }
$$

Curvilinear effects. The curvilinear nature of spherical coordinates can produce what are essentially nonlinear terms depending on how astrometric displacements are defined. These effects are on the same order as the above proper motion changes, and more pronounced near the poles. For $\mu$ as astrometry, it suffices to handle these effects by employing a rectilinear grid.

In this work, astrometric displacements labeled $\Delta \alpha \cos \delta$ and $\Delta \delta$ do not strictly refer to changes in the right ascension and declination of the star, but refer to displacements along rectilinear axes along those dimensions at the nominal position of the star. That is, if the unit vector pointing to the nominal position of the star from the solar system barycenter is defined

$$
\hat{p}=[\cos \alpha \cos \delta, \sin \alpha \cos \delta, \sin \delta],
$$

then the unit vectors pointing east and north are given by

$$
\begin{gathered}
\hat{\alpha}=[0,0,1] \times \hat{p} \\
\hat{\delta}=\hat{p} \times \hat{\alpha}
\end{gathered}
$$

and the astrometric displacements are given by

$$
\Delta \delta \equiv\left(\hat{p}^{\prime}-\hat{p}\right) \cdot \hat{\delta}
$$

$$
\Delta \alpha \cos \delta \equiv\left(\hat{p}^{\prime}-\hat{p}\right) \cdot \hat{\alpha},
$$

where $\hat{p}^{\prime}$ is the unit vector pointing to the displaced position of the star from the solar system barycenter, and $\hat{\delta}$ and $\hat{\alpha}$ are constant.

Interferometric cross terms. The one-dimensional interferometric measurement of astrometric displacement on the sky may be complicated by the motion of the reference and target stars. That is, the calculation of $\theta$ in Equation (65) may require proper motion advanced or parallax-corrected positions in a manner specific to the details of a particular instrument's measurement of $\theta$. These cross terms are likely to be small, and so they can be estimated and refined in the same manner as the other nonlinear terms, if necessary.

$R V$-astrometry cross terms. The RV semiamplitude $K$ is related to the astrometric semimajor axis, $a$, by the parallax, and so $\varpi$ is not strictly a linear parameter in the combined astrometry-RV problem. This effect can be large if the parallax is small or not known, and should not be ignored for any system.

Relativistic terms. Gravitational deflection by solar system objects and relativistic stellar aberration produce large, timedependent astrometric displacements that depend on the position of the star, and are therefore slightly nonlinear. Because these displacements can be calculated to better than $\mu$ as precision given an estimate of the star's true position to arcsecond precision, these effects are ignored here.

\subsection{Incorporating Nonlinear Terms}

Implementing these small nonlinear effects in our model requires good estimates of the astrometric parameters (when astrometric data are available, these parameters can be estimated from a first-pass solution assuming no planetary companions). The system is then solved using these estimates to calculate the second-order terms above. Below, we indicate these estimated astrometric parameters with a tilde to distinguish them from the solved parameters. These estimates can then be iteratively refined if necessary, but convergence should be very fast for SIM Lite data.

We can include these nonlinear terms by making the following substitutions to the bottom three rows of the matrix $\mathbf{F}$ in Equations (58) and (76):

$$
\begin{gathered}
\tau_{k}-t_{0} \rightarrow\left(1-\left(\tau_{k}-t_{0}\right) \frac{v_{r}}{2 \tilde{D}}\right)\left(\tau_{k}-t_{0}\right) \\
\Pi_{\alpha, k} \rightarrow\left(1-\left(\tau_{k}-t_{0}\right) \frac{v_{r}}{\tilde{D}}\right) \Pi_{\alpha, k} \\
\Pi_{\delta, k} \rightarrow\left(1-\left(\tau_{k}-t_{0}\right) \frac{v_{r}}{\tilde{D}}\right) \Pi_{\delta, k}
\end{gathered}
$$

and in the definitions of $\Pi_{k}$ (Equations (53) and (54)):

$$
\Pi_{\alpha, k} \rightarrow \Pi_{\alpha, k}+\left(\tilde{\Delta \alpha_{0}} \cos \delta+\left(\tau_{k}-t_{0}\right) \tilde{\mu_{\alpha}}\right) \vec{r} \cdot \hat{p}
$$

$$
\Pi_{\delta, k} \rightarrow \Pi_{\delta, k}+\left(\tilde{\Delta \delta_{0}}+\left(\tau_{k}-t_{0}\right) \tilde{\mu_{\delta}}\right) \vec{r} \cdot \hat{p}
$$

where the quantities in parentheses have units of radians. The estimated terms $\Delta \tilde{\alpha}_{0} \cos \delta$ and $\Delta \tilde{\delta}_{0}$ will likely be zero at first, but may be iteratively refined with the other estimated parameters.

Finally, the secular acceleration can be accommodated by simply subtracting off the appropriate, approximate linear trend to the RV data, or, if the trend parameter is present, allowing it to be absorbed in $d$.

This procedure of estimating and refining linear astrometric terms will also work for the nonlinear term introduced by the appearance of $\varpi$ in $H$ and $C$ in Equations (72) and (73). Alternatively, the parallax can be treated as a nonlinear parameter from the outset and eliminated from the linear coefficients in $\vec{\beta}$ entirely.

\section{IMPLEMENTATION}

\subsection{Public Code}

We have implemented these algorithms into a set of IDL software routines which we have made available publicly for the fitting of RV data. ${ }^{16}$ The software package includes RVLIN, the user-defined function that may be passed to MPFIT, and RV_FIT_MP, a "wrapper" routine that employs MPFIT to fit a multiplanet Keplerian model to a user-supplied set of RV data. We anticipate maintaining and improving this package, and eventually incorporating astrometric and transit data analysis. This code, or components of it, is currently used by members of the California Planet Search. Below, we discuss our software package's performance.

\footnotetext{
16 Available at http://exoplanets.org/code/
} 
Table 2

Summary of Linearization Schemes

\begin{tabular}{|c|c|c|c|}
\hline & Radial Velocities & Astrometry & $\begin{array}{l}\text { Radial Velocities } \\
\text { and Astrometry }\end{array}$ \\
\hline $\begin{array}{l}\text { Inputs } \\
\text { Nonlinear parameters }\end{array}$ & $\begin{array}{l}\vec{v}(\vec{t}), \tilde{\mu}, \tilde{\varpi} \\
P_{j}, t_{\mathrm{p}, j}, e_{j}\end{array}$ & $\begin{array}{c}\overrightarrow{\Delta \alpha}(\vec{\tau}), \overrightarrow{\Delta \delta}(\vec{\tau}), \tilde{\mu_{\alpha}}, \tilde{\mu_{\delta}}, v_{r}, \tilde{\varpi} \\
P_{j}, t_{\mathrm{p}, j}, e_{j}\end{array}$ & $\begin{array}{c}\vec{v}(\vec{t}), \vec{\rho}_{\vec{\theta}}(\vec{\tau}), \tilde{\mu_{\alpha}}, \tilde{\mu_{\delta}}, v_{r}, \tilde{\varpi} \\
P_{j}, t_{\mathrm{p}, j}, e_{j}, \Omega_{j}, i_{j}\end{array}$ \\
\hline $\begin{array}{l}\text { Transformed linear parameters } \\
\text { Linear parameters }\end{array}$ & $\begin{array}{c}c_{j}, h_{j}, v_{0} \rightarrow \omega_{j}, K_{j}, \gamma \\
d\end{array}$ & $\begin{array}{c}A_{j}, B_{j}, F_{j}, G_{j} \rightarrow \omega_{j}, \Omega_{j}, i_{j}, a_{j} \\
\Delta \delta_{0}, \Delta \alpha_{0}, \mu_{\delta}, \mu_{\alpha}, \varpi\end{array}$ & $\begin{array}{c}C_{j}, H_{j}, v_{0} \rightarrow \omega_{j}, \frac{m_{j}^{3}}{\left(m_{*}+m_{j}\right)^{2}}, \gamma \\
d, \Delta \delta_{0}, \Delta \alpha_{0}, \mu_{\delta}, \mu_{\alpha}, \varpi\end{array}$ \\
\hline
\end{tabular}

Notes. The transformed linear parameters $v_{0}$ and $\gamma$ and all of the linear parameters appear once per system. The subscript $j$ on the other parameters indicates that there are $n$ such parameters, one for each companion in the system.

\subsection{Speed-up from the Use of Explicit Derivatives}

The use of explicit derivatives above in an LM code significantly speeds up the algorithm by avoiding unnecessary calculation of numerical derivatives. To quantify this improvement, we tested two cases with published RV data, the two-planet system HD 217107 (Vogt et al. 2005) and the five-planet system 55 Cnc (Fischer et al. 2008; in both cases assuming no jitter). We combined the Lick and Keck RV data sets for both cases, solving for all $5 n+2$ parameters (including the RV offset between the two telescopes). We ran over 2500 trials, where in each trial we started the search with different initial guesses for the orbital elements, each randomly drawn from a normal distribution with a width given by the uncertainty in each parameter and centered on its best-fit value. ${ }^{17}$ We compared the total time taken for these trials on a $2.6 \mathrm{GHz}$ Intel Core 2 Duo MacBook Pro running IDL 7.0 using explicit derivatives to the time taken on the same machine with same initial guesses using numerical derivatives.

In the case of HD 217107, the use of explicit derivatives sped up the calculation by a factor of 2.3. The improvement in the case of $55 \mathrm{Cnc}$ was even larger, a factor of 4 . The total number of steps taken by MPFIT to converge on a solution was similar in the cases with and without explicit derivatives, indicating that our step sizes for the numeric derivatives were well chosen.

\subsection{Convergence Benefits of Exploiting Linear Parameters}

We employed a custom version of the multiplanet fitting routine often employed by the California and Carnegie Planet search as a baseline to test the improvement in convergence of the algorithm described in this work. This routine, which derives from the code described in Marcy \& Butler (1992) and Valenti et al. (1995), is essentially an LM algorithm for searching all $5 n+1$ parameters with carefully chosen step sizes for numerical derivatives. $^{18}$

Using the same hardware described above, we again fit the Fischer et al. (2008) $55 \mathrm{Cnc}$ RV data from two telescopes. We drew the initial guesses for every parameter from normal distributions centered on the Keplerian best-fit values and with width given by $s \sigma_{x}$, where $\sigma_{x}$ is the parameter uncertainty quoted in Fischer et al. (2008) and $s$ is a scale factor. We varied $s$ smoothly from 0 (where the initial guesses were the best-fit values exactly) to 10 (where every parameter is independently, randomly altered by $10 \sigma_{x}$ ) over 4000 trials.

\footnotetext{
17 Although dynamical fits are more precise, here we are only concerned with the algorithm's convergence in the region of the global $\chi^{2}$ minimum for a purely Keplerian fit. The initial guesses for the parameters in this and the test in Section 5.3 were thus drawn near their values at this (presumed) global minimum.

18 That is to say, the only significant differences between the baseline code and the code described in Section 5.1 is the exploitation of linear parameters.
}

We did not attempt to fit for the telescope offset at each trial, as this particular routine was not optimized for such a task. We also did not vary the $\gamma$ parameter from its best-fit value (though we did fit for it), as no uncertainties were given for $\gamma$ in Fischer et al. (2008).

We compared the results of this custom code to our LM code employing linear parameters (Section 5.1) with both explicit and numerical derivatives. In some sense, this is not a fair test since the linear parameter models do not require or accept initial guesses for $K$ and $\omega$, and thus the initial guesses are closer to the best-fit values (since they differ in only 15 space dimensions, whereas the guesses for the nonlinear routine differ in 25 dimensions). We thus also ran an additional test, where the fully nonlinear routine was provided the best-fit values for $K$ and $\omega$ for each planet, and thus had only 15 parameters varied (but still had all 26 parameters to fit). With this exception, we provided the same initial guesses to each of the four schemes in each trial.

We recorded the final $\chi^{2}$ reported by each routine for each trial and compared this with $\chi_{\min }^{2}$, the best-fit value. We deemed any trial for which $\left(\chi^{2}-\chi_{\min }^{2}\right)<2$ to be a "successful" convergence on the correct parameters. ${ }^{19}$ At each tested value of $s$, we weighted the full set of trials by a Gaussian window 75 trials wide (corresponding to $\sim 0.19 \sigma$ ) and calculated the fraction on trials that were successful.

In Figure 1, we plot the fraction of successful tests as a function of $s$. The use of numerical derivatives had no overall effect on the convergence of the linear parameter routine, but was slower, on average, by a factor of 4 .

Even with initial guesses $10 \sigma_{x}$ from the best-fit values in all 15 parameters, the linear parameter routine found the global minimum of $\chi^{2}$ in roughly half of all trials. The fully nonlinear routine, searching a 26-dimensional space, required much better initial guesses to achieve convergence. With the same 15 parameters varied, fits with initial parameters off by $3 \sigma_{x}$ had only a $20 \%$ chance of properly converging. With all 25 orbital parameters varied, the initial guesses had to be within $1.5 \sigma_{x}$ of their proper value to have a $50 \%$ chance of convergence. With 26 dimensions to search, there are many wrong paths for the LM algorithm to follow away from the global $\chi^{2}$ minimum.

\section{CONCLUSIONS}

Applying linear parameters to the problem of fitting Keplerian curves to RV and astrometry data significantly improves the efficiency and reliability of multi-planet Keplerian fitting routines. This technique can be applied to many methods

\footnotetext{
$19 \chi_{\min }^{2} \sim 2910$. Our results are only weakly sensitive to the precise definition of "successful."
} 


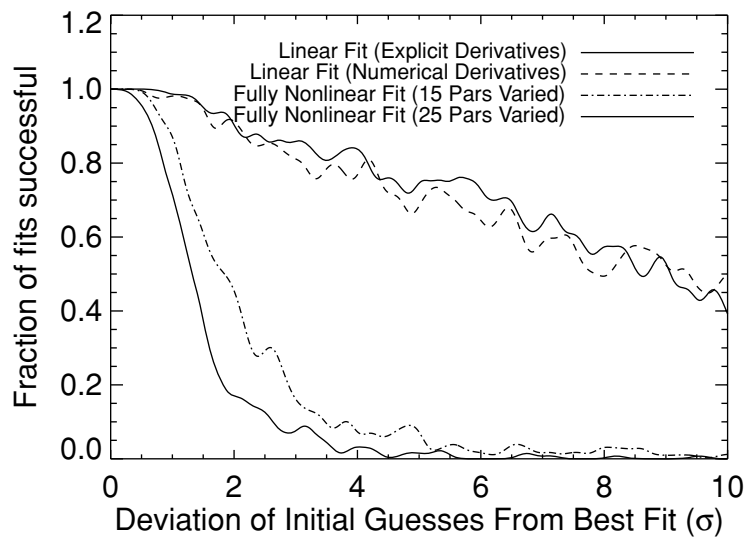

Figure 1. Sensitivity of RV-only fitting algorithms to initial guesses on the fiveplanet, two-telescope RV data for 55 Cnc (Fischer et al. 2008). Initial guesses to the four fitting routines were randomly varied from their best-fit values by various factors of their respective uncertainties. The linear parameter fitting algorithm described in this work converged on the best solution for a wide range of initial guesses, including $\sim 50 \%$ of the cases with guesses $10 \sigma$ from nominal. A full, 26-parameter nonlinear fit required guesses within $1.5 \sigma$ of the best-fit value, or within $2 \sigma$ if only the 15 truly nonlinear parameters were varied.

of searching this complex, nonlinear, multiparameter $\chi^{2}$ space, including the LM method, MCMC algorithms, and brute force approaches. Table 2 summarizes the various schemes used in this work.

We have identified the nonlinear terms relevant for $\mu$ as astrometry and $\mathrm{m} \mathrm{s}^{-1} \mathrm{RV}$ work (such as that by SIM Lite and its supporting RV data), and shown how to incorporate these terms into a linear parameter scheme. In Section 2.4, we have provided analytic forms for explicit derivatives relevant to various applications of the Kepler problem.

In the case of RV-only data, the use of explicit derivatives can speed up a fitting routine by a factor of $2-4$, depending on the number of planets being fit. The use of linear parameters greatly improves the convergence properties of a multiplanet fitting routine. In the case of an actual five-planet fit, a linear parameter model requires initial guesses within $10 \sigma_{x}$ of their correct values in only 15 parameters to have a $50 \%$ chance of convergence, while a full, 26-parameter search requires all 26 parameters to be specified within $1.5 \sigma_{x}$.

The principal improvement from use of linear parameters comes from reducing the search space for an $n$-planet model. In the case of RV fitting, the reduction is from exploiting the linearity of $2 n+1$ of the $5 n+1$ fitted parameters, leaving only $3 n$ nonlinear parameters to be fit algorithmically. The problem of fitting astrometric orbits can be similarly treated by exploiting the linearity of $4 n+5$ of the $7 n+5$ parameters, leaving, again, only $3 n$ nonlinear parameters to be with a nonlinear fitting routine. When combining RV and astrometric data, as will be the case for SIM Lite, only $2 n+6$ of the $7 n+6$ model parameters are usefully linear under the scheme described here, leaving $5 n$ nonlinear parameters.

We have derived a general expression for the explicit model derivatives for models employing linear parameters in nonlinear fits, appropriate for application in the LM method. This result is general and can be applied to problems beyond Keplerian fitting-indeed to any model with both linear and nonlinear parameters.
Eric Agol inspired Section 2 by pointing out how to exploit the linear parameters in the problem of RV fitting in the context of MPFIT. He has provided substantial guidance and ideas for this and future versions of our code. We thank him, Eric Ford, Matthew Muterspaugh, Tom Loredoo, and Alessandro Sozzetti for careful readings of this manuscript and their many substantive and constructive suggestions.

Our membership on a SIM Planet-Finding Astrometry Analysis Team (P.I.: Matthew Muterspaugh) motivated Sections 3 and 4 of this work, and we are grateful to all of the teams involved for their efforts and insights into the problem. We thank Jeff Valenti and Nikolai Piskunov for encouraging us to calculate the explicit derivatives for a general linear least-squares problem. Nikolai Piskunov provided insight into the sources of instability in the LM search algorithm. Matthew Muterspaugh provided guidance with the problem of fitting astrometric orbits and understanding the small astrometric cross terms. We thank Martin Sirk, Sam Halverson, and John Asher Johnson for their work employing and debugging various versions of our code. J.T.W. received support from NSF grant AST-0504874, and A.W.H. received support from NASA contract NAS7-03001 (JPL\#1336910).

\section{REFERENCES}

Bakos, G. Á., et al. 2009, arXiv:0901.0282v1

Balan, S. T., \& Lahav, O. 2008, MNRAS, 394, 1945

Binnendijk, L. 1960, Properties of Double Stars: A Survey of Parallaxes and Orbits (Philadelphia, PA: Univ. Pennsylvania Press)

Butler, R. P., et al. 2006, ApJ, 646, 505

Casertano, S., et al. 2008, A\&A, 482, 699

Catanzarite, J., Law, N., \& Shao, M. 2008, in SPIE Conf. Ser. 7013, Astrometric Detection of Exo-Earths in the Presence of Stellar Noise, (Bellingham, WA: SPIE)

Catanzarite, J., Zhai, C., \& Shao, M. 2009, in AAS Meeting Abstracts 213, 456.03

Cumming, A., Marcy, G. W., Butler, R. P., \& Vogt, S. S. 2003, in ASP Conf. Ser. 294, Scientific Frontiers in Research on Extrasolar Planets (San Francisco, CA: ASP), 27

Driscoll, P., \& Fischer, D. 2005, BAAS, 37, 1269

Eriksson, U., \& Lindegren, L. 2007, A\&A, 476, 1389

Fischer, D. A., et al. 2008, ApJ, 675, 790

Ford, E. B. 2004, in AIP Conf. Ser. 713, The Search for Other Worlds, ed. S. S. Holt \& D. Deming (New York: AIP), 27-30

Ford, E. B. 2008, AJ, 135, 1008

Hastings, W. 1970, Biometrika, 57, 97

Konacki, M., \& Maciejewski, A. J. 1999, ApJ, 518, 442

Konacki, M., Maciejewski, A. J., \& Wolszczan, A. 2002, ApJ, 567, 566

Levenberg, K. 1944, Quart. Appl. Math., 2, 164

Marcy, G. W., \& Butler, R. P. 1992, PASP, 104, 270

Markwardt, C. B. 2009, arXiv:0902.2850

Marquardt, D. 1963, SIAM J. Appl. Math., 11, 431

Metropolis, N., Rosenbluth, A. W., Rosenbluth, M. N., Teller, A. H., \& Teller, E. 1953, J. Chem. Phys., 21, 1087

Press, W. H., Teukolsky, S. A., Vetterling, W. T., \& Flannery, B. P. 1992 Numerical Recipes in FORTRAN: The Art of Scientific Computing (2nd ed.; Cambridge: Cambridge Univ. Press)

Scargle, J. D. 1982, ApJ, 263, 835

Seidelman, P. K. (ed.) 1992, Explanatory Supplement to the Astronomical Almanac (Nautical Almanac Office, U.S. Naval Observatory/H. M. Nautical Almanac Office, Royal Greenwich Observatory, et al.) (Mill Valley, CA: University Science Books)

Sozzetti, A. 2005, PASP, 117, 1021

Valenti, J. A., Butler, R. P., \& Marcy, G. W. 1995, PASP, 107, 966

Vogt, S. S., Butler, R. P., Marcy, G. W., Fischer, D. A., Henry, G. W., Laughlin, G., Wright, J. T., \& Johnson, J. A. 2005, ApJ, 632, 638

Wright, J. T., Upadhyay, S., Marcy, G. W., Fischer, D. A., Ford, E. B., \& Johnson, J. A. 2009, ApJ, 693, 1084

Wright, J. T., et al. 2007, ApJ, 657, 533 


\title{
ERRATUM: "EFFICIENT FITTING OF MULTI-PLANET KEPLERIAN MODELS TO RADIAL VELOCITY AND ASTROMETRY DATA" (2009, ApJS, 182, 205)
}

\author{
J. T. WrighT ${ }^{1}$ AND A. W. HowARD ${ }^{2}$ \\ 1525 Davey Laboratory, Center for Exoplanets and Habitable Worlds, Department of Astronomy and Astrophysics, \\ The Pennsylvania State University, University Park, PA 16802, USA; jtwright@astro.psu.edu \\ 2 Institute for Astronomy, University of Hawaii, 2680 Woodlawn Drive, Honolulu, HI 96822, USA \\ Received 2013 February 21; published 2013 April 3
}

We correct a mathematical and descriptive error in our manuscript. When radial velocity measurements of stars are made to infer planets' orbits, it is the argument of periastron $(\omega)$ of the star that is reported, since that is the observed quantity. Our derivation incorrectly describes $\omega$ in this case as that of the planet. The conclusions and methodology described are correct, as is the output of the RVLIN package described in the published paper. Three equations (which might be used in a practical implementation of the algorithm) should be changed.

When radial velocity measurements of stars are made to infer planets' orbits, it is the argument of periastron of the star $(\omega)$ that is usually reported in the literature, since that is the observed quantity. Because the motion of a star due to a single orbiting planet is a point reflection of the planet's motion, the parameters $P, e, t_{\mathrm{p}}, i$, and $\Omega$ and the anomalies $f, E$, and $M$ are identical for the star and planet in the one-planet case.

In Table 1 we define $\omega$ (and, by extension, $\omega_{j}$ ) to be the argument of periastron of the orbit of the planet about the center of mass of the system. The paper is most easily corrected by reinterpreting all instances of $\omega_{j}$ as referring to the portion of the star's motion due to the influence of the $j$ th planet (which, in the non-interacting approximation of the paper, is purely Keplerian motion, i.e., an ellipse.)

Only Equations (69), (72), and (73) need to be modified with this understanding; all other relations and algorithmic descriptions become correct.

These are the instances in the paper that should be ignored or modified for clarity, consistency, and correctness:

1. Table 1 should specify that all instances of $\omega$ refer to the motion of the star.

2. The first appearance of $\omega$ at the beginning of Section 2.1 should specify that this refers to the orbit of the star.

3. The description of orbital parameters in the last paragraph of p. 206 state that they are "the usual Keplerian parameters for the planet $j$," when in fact they are for the portion of the star's reflex motion due to planet $j$.

4. Footnote 10 and its referring text imply that $\omega_{*}$ differs from $\omega$ and $\omega_{j}$ elsewhere in the text, which is incorrect.

5. The sentence after footnote 10 could be clarified to read “...the astrometric elements of the components of the star's motion about the center of mass of the system due to the planet"

6. Equation (47) is no longer correct and should be ignored; footnote 10 clarifies this point.

7. At the top of the second column on p. 210, there is no need to distinguish $\omega_{*}$ from $\omega$.

8. Equations (69), (72), and (73) should read

$$
\tan \omega_{j}=\frac{C_{j}}{H_{j}}
$$

where $\omega_{j}$ is chosen so $\sin \omega_{j}$ has the same sign as $C_{j}$.

$$
\begin{aligned}
& h_{j}=\lambda_{j} H_{j} \\
& c_{j}=-\lambda_{j} C_{j}
\end{aligned}
$$

In addition, the description of the difficulties of using Lagrange multipliers on p. 211 is ill posed, and should be ignored.

Because the public code RVLIN employs only the radial velocity component of the star's motion, it is unaffected by these changes and produces correct output, provided one interprets that output as referring to the star's motion, not the planet's. Any implementation of the full astrometric plus RV algorithm must employ the proper version of Equations (69), (72), and (73).

The authors thank B. Scott Gaudi for bringing this issue to their attention. 